Graduate Students

Health and Biomedical Sciences

\title{
Influenza Prevalence and Vaccine Efficacy in Diabetic Patients in Qatar Swapna Thomas ${ }^{1}$, Maria K. Smatti ${ }^{1}$, Muna A. Al Maslamani ${ }^{2}$, Hadi M. Yassine ${ }^{1 *}$ \\ ${ }^{1}$ Qatar University, ${ }^{2}$ Hamad Medical Corporation
}

\section{Background}

Diabetes (DM) is often recognized as an independent risk factor for developing severe respiratory tract infections. Influenza infections are associated with higher hospitalization, morbidity and mortality in DM patients. Vaccination against Influenza virus would reduce the burden of flu infection in DM patients.

\section{Research Aim}

To assess the prevalence and burden of influenza infection in DM patients in Qatar and to evaluate the efficacy of influenza vaccination in DM patients

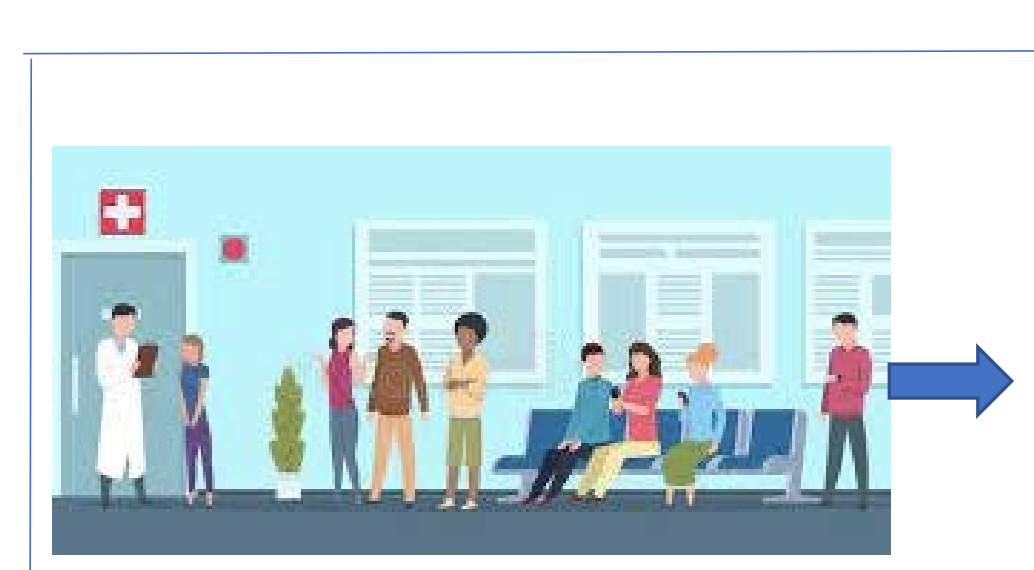

26989 participants Year $2016-2018$

\section{Methodology}

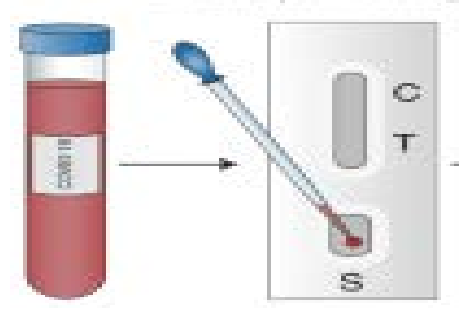

Virology tests 20 Pathogens

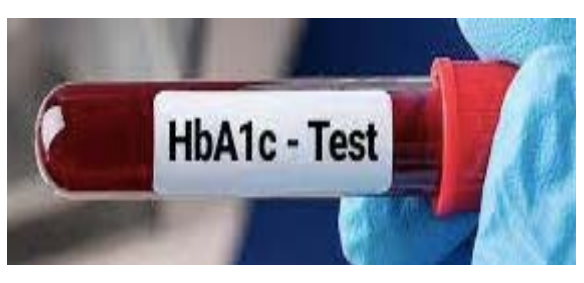

HbA1c test

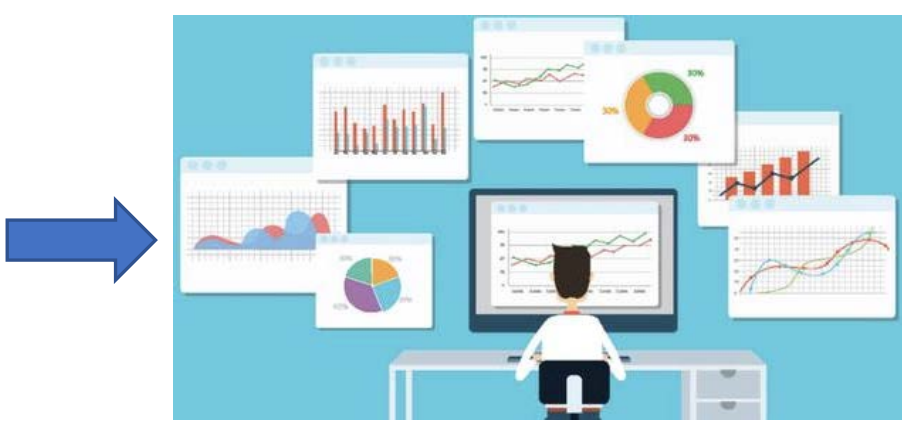

Statistical Analysis

\begin{tabular}{|c|c|c|c|c|}
\hline \multicolumn{7}{|c|}{ Summary of Study Participants } \\
\hline DM & Non-DM & Vaccinated & Non-Vaccinated & Total \\
\hline 2740 & 23949 & 2182 & 558 & 26989 \\
\hline
\end{tabular}

\section{Findings}

1. Etiology of respiratory infections in DM and Non-DM patients

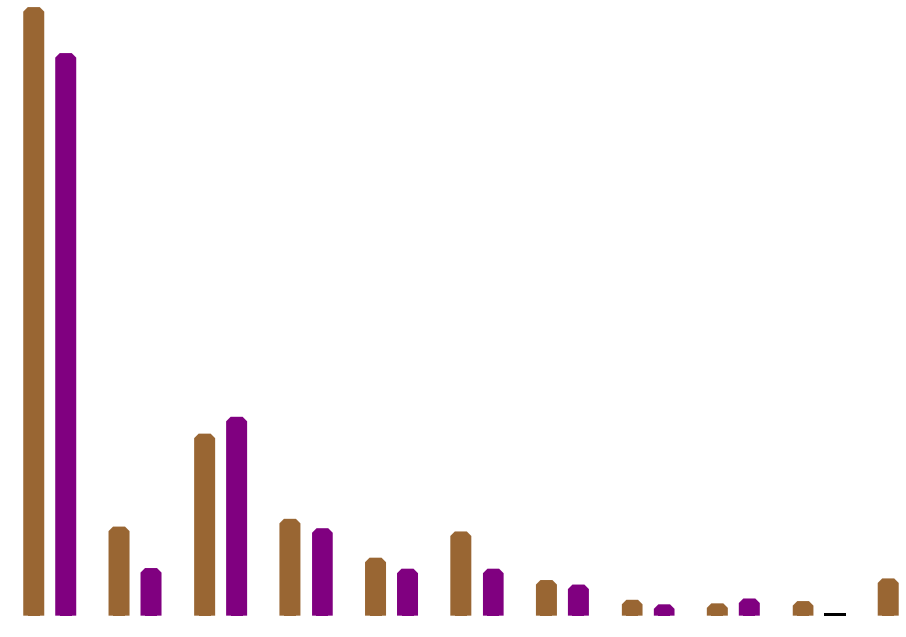

2. Effect of Influenza Vaccination in DM patients

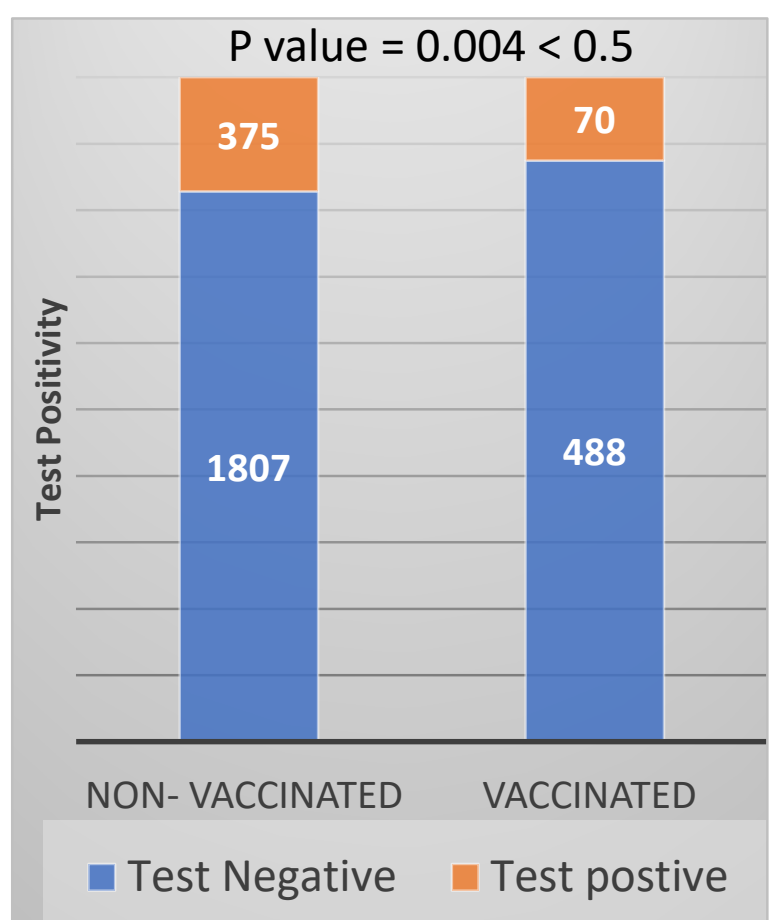

3. Clinical symptoms in vaccinated and non-vaccinated DM patients

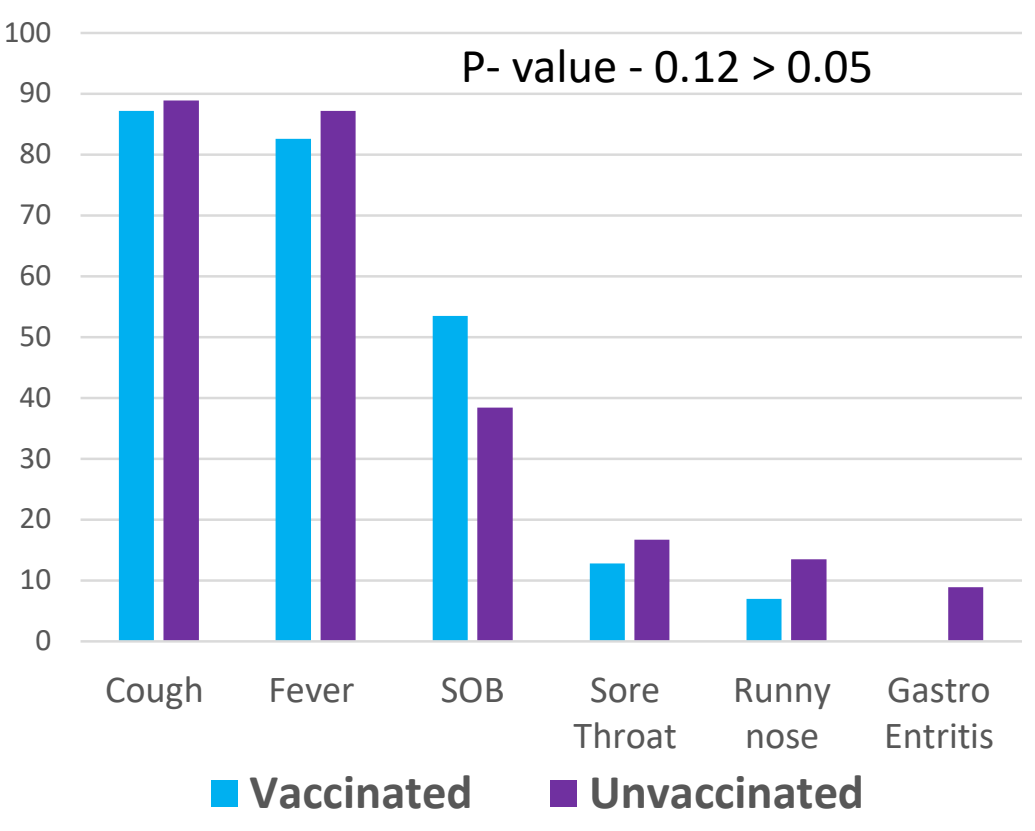

\section{Conclusion}

$>\quad$ DM patients are at higher-risk for influenza infection

$>\quad$ Influenza infections covers around $50 \%$ of total respiratory infections in patients with flu like symptoms in Qatar In vaccinated DM patients, influenza test positivity is low.

This study reports the efficacy of routine flu vaccination to reduce the burden of influenza infection in DM patients. 